\title{
Design and Application of Intelligent Building Control System
}

\section{Chaoguo Ma}

Xinjiang Haochen Architectural Planning and Design Institute, Changji, Xinjiang, 831100, China

\begin{abstract}
The information age has promoted the further development of building intelligence. People will use the intelligent control system more to make the building more intelligent and information, which is the benefit of the development of the times to the construction industry, in the future, the building will rely on the intelligent system to continue to develop. This paper mainly analyzes the design and application of intelligent building control system, first defines the intelligent building, then analyzes the design of intelligent system, and finally analyzes the application of intelligent building system.
\end{abstract}

\section{Keywords}

building intelligence; system design; engineering application

\section{建筑智能化控制系统设计与应用}

\section{马朝国}

新疆昊辰建筑规划设计研究院有限公司, 中国・新疆 昌吉 831100

\section{摘 要}

信息时代有力地推动着建筑智能化的进一步发展。人们将智能化的控制系统更多地运用在建筑方面，使建筑更加智能化、信 息化, 这是时代发展给建筑行业带来的好处, 在未来建筑会依靠智能系统继续发展。论文主要分析建筑智能化控制系统设计 与应用, 首先对智能建筑下定义, 然后分析智能化系统的设计, 最后对建筑智能化系统应用进行分析。

\section{关键词}

建筑智能化; 系统设计; 工程应用

\section{1 引言}

当前人们除了重视对于饮食方面的需要, 也更加重视住 房方面的需求，希望居住与工作场所更舒适，建筑智能化系 统能很大程度地满足人们在建筑方面的需求。建筑智能化系 统也是比较复杂的, 它需要用到人工智能、自动化电气、建 筑工程、通信工程、计算机以及艺术等多学科方面的知识。 一方面展现出现代建筑艺术, 又与信息技术相结合, 是多学 科知识融合的产物。建筑智能化主要体现在智能化住宅与办 公楼方面, 提升了人们的生活质量; 另一方面建筑智能化的 飞速发展对建筑行业来说也是一个跨越。

【作者简介】马朝国（1978-）, 男, 工程师, 新疆吴辰建筑 规划设计研究院有限公司分院副院长, 从事电气自动化设计 应用研究。

\section{2 智能建筑的定义}

智能建筑主要是借助信息时代给建筑行业所带来的优势 将智能信息技术与建筑行业相结合，它利用系统集成方法把 多学科的技术相结合起来, 并且它能对建筑内的情况自动监 控, 对信息资源实现有效管理, 很大程度上减少了人力的投入。 智能建筑还可以将不同的要素相组合, 包括智能化系统要素、 管理要素、结构要素和服务要素, 要素的有效组合能给用户 提供一个比较舒适、安全、便利、人性化和高效的产品。智 能建筑还能搭上信息时代的列车, 充分满足用户需求。办公 自动化系统, 智能化的集成系统, 通信网络系统以及建筑设 备监控系统共同组成了建筑智能化系统。智能建筑如果运用 在具体的建筑工程中也需要满足两点要求, 首先它要满足用 户最基本的需求, 也就是说能给用户提供满足生活需要的环 
境, 并且建筑自身的资源要得到良好的配置, 包括建筑的运 营管理、设备运行、控制与设备维护等通讯设施，在建筑内 能与外界取得联系。此外, 智能建筑的投入也需要有一些基 本的经济产出。

\section{3 建筑智能化系统设计分析}

\section{1 设计背景分析}

建筑智能化系统的设计比较复杂, 涉及多种学科知识。 智能建筑是一个使用复合学科知识的建筑, 同样地在设计中 涉及多方面的内容，包括建筑设备、通信设备、线路布置以 及监控系统等, 所以说也对设计人员提出了比较高的要求。 设计人员自身不仅要在设计方面有比较扎实的基础知识以及 创新思维，还要掌握多方面内容的基础知识 ${ }^{[1]}$ 。除了这些内 容之外, 设计人员要遵循相关的规定与原则, 有非常高的创 新意识, 对原有的设计方法以及设计理念进行不断的创新。 建筑工程在建造的时候会使用不同的技术，设备和原材料。 设计师在设计的时候要对这些方面的内容比较熟悉。建筑工 程的原材料以及设备可能来自各地, 设计师需要对这些材料 掌握得比较清楚, 以便进一步调整设计内容, 减少在建筑过 程中可能会产生的问题, 充分发挥建筑智能系统设计的作用。

\section{2 建筑智能化控制系统的构成和结构设计分析}

CAN 总线属于现场总线的范畴，是一种有效支持分布式 控制或实时控制的串行通信网络。从原理和实现的角度, 只 要有两个 CAN 节点和通讯介质就可以构成一个 CAN 总线系 统。多个 CAN 节点可以构成较大的 CAN 总线系统。CAN 节 点按照不同的功能又可以分为数据采集节点、传感器节点、 执行器节点、监控节点等。CAN 总线系统与上位机连接, 进 而可以构成一个更大的网络系统 ${ }^{[2]}$ 。论文所介绍的建筑智能 化控制系统正是基于 CAN 总线的原理而设计和开发的, 它将 分散的、能完成多种功能的模块设备, 通过简单的总线连接, 进而完成比较复杂的系统功能。模块设备之间可以进行信息 交换。不同功能的模块设备能以不同的方式与外界环境或外 部设备进行信息交换：或采集外界环境信息，或向外部设备 输出系统的指令要求。系统可以对现场终端进行实时监控和 管理。每个模块设备均内置微处理器, 运行参数被分散存储 在各个模块设备中, 即使系统断电也不会丢失, 通信速度快, 运行效率高, 见图 1 。

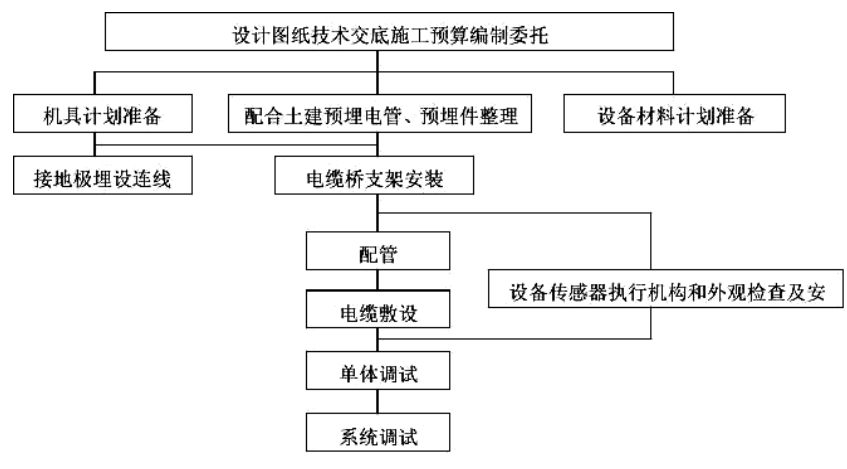

图 1 建筑智能化系统组织设计组织结构图

\section{3 建筑智能化控制系统的硬件设计与软件设计}

干路管理器和支路管理器硬件相同（软件不同），采用 64 引脚 STM32F103 系列芯片作为微处理器, 模块设备则均 采用 48 引脚 STM $32 \mathrm{~F} 103$ 系列芯片作为微处理器; 所有设备 通信均采用 CAN 总线的方式，使用 $82 \mathrm{C} 250$ 芯片; 干路管理 器和支路管理器增加了以太网的通信方式, 采用以太网芯片 ENC28J60。

各模块设备所使用其他主要芯片如下: 模拟量输入模块 使用了MAX485 芯片; 模拟量输出模块使用了 TLC5615 模 数转换芯片; 单值数字量输入模块和定时器模块使用了触摸 芯片 ST04D

软件设计由上层管理系统软件 (上位机软件) 和现场控 制系统软件两大部分构成。上位机软件可以用 VB 制作，也 可以使用组态软件。上位机软件即为上层监控管理软件, 用 以查看现场各智能节点的运行状态、参数设置、报警显示等。 现场控制系统软件用 C 语言完成, 即面向 STM32 芯片进行 编程，使用库开发的方式。不同功能的智能节点程序也不尽 相同，主要包括通讯、显示、控制、采集等功能程序，见图 2 。

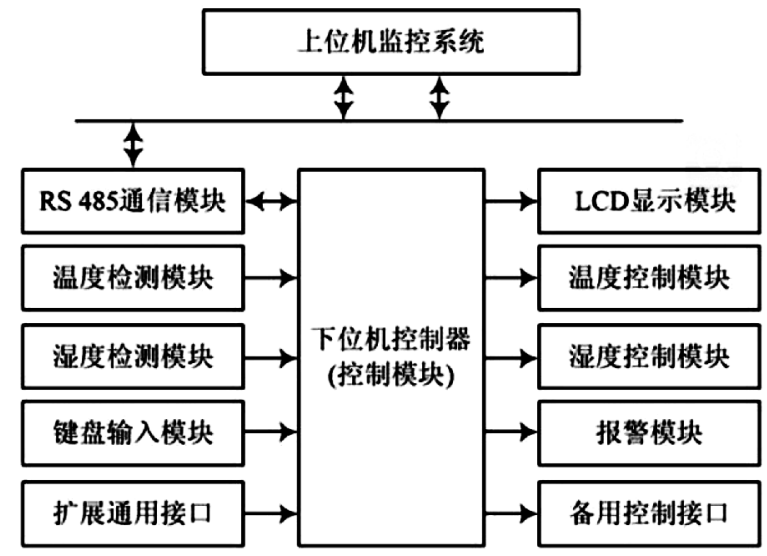

图 2 建筑智能化控制系统的硬件设计与软件设计 


\section{4 建筑智能化系统应用分析}

\section{1 多功能会议系统}

智能化的建筑系统可以非常有效地运用在办公楼当中。 当前很多地方都是现代化的办公场所, 需要至少满足办公室 音响及扩音、投影及影音、视频会议以及监控视频这四方面 的需求。为了使数据资料传输的速度更快以及传输质量更安 全, 还可以在调音室内使用一个专业的跳线实行端接。在设 计过程中设计师主要考虑使用多功能的系统设计达到智能化 的目的, 并通过智能化系统提升会议效果。

\section{2 通信智能化系统}

通信智能化系统能疏通办公室内外的通信，也可以提高 办公室的智能化。电子政务办公应用系统、计算机网络系统 和机关办公服务系统组成了办公楼的智能化设计的系统。其 中, 电子政务办公系统可以推进政府内部对企业公众的工作 发展, 计算机网络系统是自动化办公楼的核心, 通过这个网 络系统能推动计算办公楼网络应用软件的应用, 还可以收集 整理和分析各种数据信息并作出决策 ${ }^{[3]}$ 。这个系统主要是通 过内部的局域网来实现的, 它应用的速度非常快。机关办公 服务系统可以设定电锁控制车库控制点以及门禁点, 通过控 制点与门禁点完成相关的开关门车库系统的身份识别, 用户 只需要刷卡就可以完成信息识别, 非常便利。

\section{3 防火警报系统}

防火警报系统主要是由消防器械, 火灾探测器和控制器 组成。首先, 火灾探测器可以在火灾达到一定的亮度和温度时, 火灾探测器可以释放出某种可识别的型号, 将数据上传到某 种控制设备中。其次, 火灾警报控制器可以第一时间接收到
火灾探测器的型号并且能准确地做出反应与判断, 再结合消 防器械就能及时地控制火情, 减少火灾的发生。

\section{4 保安监控系统}

保安监控系统由三个相关系统组成, 分别是主被动防盗 警报系统、闭路电视监控系统和门禁系统, 这些系统的设置 能促进办公楼内的财产信息安全的保护。设置好监控点, 尤 其是地下车库的防盗系统, 它能通过智能化设置对车库的内 部的环境状况进行全面无死角的监控, 遇到问题时也能通过 监控系统查找情况, 保证车库的安全, 减少用户财产损失。

\section{5 结语}

设计人员在设计过程中需要具备专业的设计知识和多学 科的知识以及过硬的设计功底, 将智能化的信息系统充分应 用到建筑设计之中, 与时俱进，满足用户需求。作为信息时 代与建筑工程相结合的产物, 随着人们需求的发展以及时代 的不断发展, 建筑智能化系统的应用会更加广泛, 它能有效 地提升人们生活质量, 保护环境, 促进经济发展。建筑智能 化控制系统在当今注重环境保护的时代前景广阔, 并且对中 国智能化的发展有非常重要的作用。

\section{参考文献}

[1] 伍银波, 杨勇. 基于 Modbus/TCP 及 PLC 的建筑智能化系统设计 [J]. 信息技术 ,2014(06):64-66.

[2] 史建阳. 建筑智能化系统设计的分析 [J]. 新材料新装饰, 2014 (04):451.

[3] 高红红, 韩怀宝, 孙玉龙, 等. 医院建筑智能化信息设施系统设计 [J]. 现代建筑电气, 2016(06):1-5+18. 